University of Warwick institutional repository: http://go.warwick.ac.uk/wrap This paper is made available online in accordance with publisher policies. Please scroll down to view the document itself. Please refer to the repository record for this item and our policy information available from the repository home page for further information.

To see the final version of this paper please visit the publisher's website. access to the published version may require a subscription.

Author(s): Martin Golubitsky, Liejune Shiau and Ian Stewart

Article Title: Spatiotemporal symmetries in the disynaptic canal-neck projection

Year of publication:2007

Link to published version:http://dx.doi.org/ 10.1137/060667773

Publisher statement: None 


\title{
SPATIOTEMPORAL SYMMETRIES IN THE DISYNAPTIC CANAL-NECK PROJECTION*
}

\author{
MARTIN GOLUBITSKY ${ }^{\dagger}$, LIEJUNE SHIAU ${ }^{\ddagger}$, AND IAN STEWART ${ }^{\S}$
}

\begin{abstract}
The vestibular system in almost all vertebrates, and in particular in humans, controls balance by employing a set of six semicircular canals, three in each inner ear, to detect angular accelerations of the head in three mutually orthogonal coordinate planes. Signals from the canals are transmitted to eight (groups of) neck motoneurons, which activate the eight corresponding muscle groups. These signals may be either excitatory or inhibitory, depending on the direction of head acceleration. McCollum and Boyle have observed that in the cat the relevant network of neurons possesses octahedral symmetry, a structure that they deduce from the known innervation patterns (connections) from canals to muscles. We rederive the octahedral symmetry from mathematical features of the probable network architecture, and model the movement of the head in response to the activation patterns of the muscles concerned. We assume that connections between neck muscles can be modeled by a "coupled cell network," a system of coupled ODEs whose variables correspond to the eight muscles, and that this network also has octahedral symmetry. The network and its symmetries imply that these ODEs must be equivariant under a suitable action of the octahedral group. It is observed that muscle motoneurons form natural "push-pull pairs" in which, for given movements of the head, one neuron produces an excitatory signal, whereas the other produces an inhibitory signal. By incorporating this feature into the mathematics in a natural way, we are led to a model in which the octahedral group acts by signed permutations on muscle motoneurons. We show that with the appropriate group actions, there are six possible spatiotemporal patterns of time-periodic states that can arise by Hopf bifurcation from an equilibrium representing an immobile head. Here we use results of Ashwin and Podvigina. Counting conjugate states, whose physiological interpretations can have significantly different features, there are 15 patterns of periodic oscillation, not counting left-right reflections or time-reversals as being different. We interpret these patterns as motions of the head, and note that all six types of pattern appear to correspond to natural head motions.
\end{abstract}

Key words. vestibular system, Hopf bifurcation, spatiotemporal symmetries, coupled cell systems

AMS subject classifications. 92C20, 37G40, 34C25

DOI. $10.1137 / 060667773$

1. Introduction. The human vestibular system is a system of tubes that contain sensors for motion and orientation in space, yielding the sense of balance. There are two main components: the otolith organs, which sense linear acceleration of the head (translation), and the semicircular canals, which sense angular acceleration of the head (rotation). Each ear contains three semicircular canals (henceforth "canals") arranged in three approximately mutually orthogonal planes; see Figure 1 below. A similar arrangement occurs in most vertebrates. We do not discuss the otolith system or other physiological features of the sense of balance.

In this paper we focus on two points. First, we rederive the symmetry group $\Gamma$ of the network of neurons that conveys signals from the six canals to eight principal

\footnotetext{
* Received by the editors August 18, 2006; accepted for publication (in revised form) March 14, 2007; published electronically July 20, 2007. This work was supported in part by NSF grants DMS0244529 and DMS-0604429.

http://www.siam.org/journals/siap/67-5/66777.html

${ }^{\dagger}$ Department of Mathematics, University of Houston, Houston, TX 77204-3008 (mg@uh.edu).

${ }^{\ddagger}$ Department of Mathematics, University of Houston-Clear Lake, Houston, TX 77058 (shiau@cl. uh.edu).

$\S$ Mathematics Institute, University of Warwick, Coventry CV4 7AL, UK (ins@maths.warwick.ac. uk). The work of this author was supported in part by a grant from EPSRC.
} 
muscle groups that control the position of the neck. McCollum and Boyle [12] analyzed experimental work of Shinoda et al. [13, 14, 15] and Wilson and Maeda [16] to discover these symmetries. Our derivation makes transparent the fact that $\Gamma$ is the 48 -element symmetry group of the cube, which is called the octahedral group. This network of connections is known as the canal-neck projection.

Second, we assume that the octahedral group $\Gamma$ is also the symmetry group of the internal dynamics of the muscles and associated neural connections, and we use these symmetries to discuss natural rhythmic head motions. We look only for small amplitude periodic head motions that can be sustained by the neck muscles alone. In particular, we assume that the sensory inputs from the canals are not relevant, except to prescribe the symmetries of the system. A similar approach has been applied previously to spatiotemporal patterns in animal locomotion; see Buono and Golubitsky [3], Collins and Stewart $[4,5]$, and Golubitsky et al. [10, 11]. However, in those papers the patterns of locomotion were used to infer the symmetry of the network of neurons (central pattern generator) that produced them, whereas here we infer the patterns of movement from the known symmetries of the canal-neck projection.

Our approach is straightforward but not completely standard. The work of McCollum and Boyle [12] suggests a simplest network for the motoneurons of the eight muscle groups. Although we do not know (and perhaps cannot know) an accurate differential equation model for the (abstracted) muscle motoneurons, we can presume the form that such a model will take. We use the symmetries and the network structure to answer the question: What are the spatiotemporal symmetries of small amplitude periodic solutions that can be obtained by Hopf bifurcation from a group invariant equilibrium in this class of possible models? (These periodic solutions are the ones that can most naturally exist in models near a position where the head is held fixed and upright. A more general classification of the spatiotemporal symmetries of periodic solutions, whose amplitudes are not necessarily small, can be made using the $H / K$ Theorem $[3,9]$. However, we choose to begin our classification with the more restricted problem of small amplitude periodic solutions near an upright head.)

Using a caricature of the physical actions of the muscle groups, we observe that a group invariant equilibrium corresponds to one in which the head is held fixed. Using this caricature, we can also interpret the form that the head motions will take based only on the spatiotemporal symmetries of the associated periodic solutions. In this sense our approach is model-independent; it does not depend on the particular system of ODEs. Our results provide a list, or menu, of the possible head motion types; specific models and specific parameters in the models choose from this menu and determine which solution types exist and which are stable. We do not discuss such model-dependent issues here.

In order to relate these spatiotemporal symmetries to characteristic head motions, we need to make assumptions about how the eight muscle groups move the head. For physiological and mathematical reasons we are led to classify the eight muscle groups into four opposing pairs. When both muscles in a pair are equally activated the head will not move. Indeed, to move the head, one muscle group must pull harder than the opposing one; we classify only those periodic states that satisfy this constraint.

To analyze the possible dynamics we employ the theory of dynamical systems with symmetry, which has implications for the dynamics of such a network. We restrict ourselves to classifying those types of head motions that can be described by small amplitude periodic states near a group invariant equilibrium. The mathematical tool for performing this classification is the equivariant Hopf bifurcation theorem [8, 9]. In particular, we use the results of Ashwin and Podvigina [1] on Hopf bifurcation with 
octahedral symmetry.

This classification is "model-independent" in the sense that it does not depend upon the detailed structure of the network of neurons concerned, or on the precise equations used to model neurons, provided that the symmetry constraints are respected. Since all model equations in current use are primarily phenomenological, and the precise architecture of the muscle group network is unknown (even in the cat), model-independent results have a potential advantage: they depend only on the known symmetries of the network. Any specific choice of network architecture and model neuron dynamics (associated, for example, with particular vertebrate species) will generate a list of spatiotemporal patterns taken from the general classification, but with extra model-dependent restrictions on existence and stability. The modelindependent features of the problem can also help to structure existence and stability calculations in specific models; see [9].

In order to create this menu and to make predictions about head motions, we must determine the appropriate "phase space" variables upon which the group $\Gamma$ acts, and also specify the appropriate group action. Our approach, as in the gaits work, is to use the network structure. We assume that each of the eight motoneurons (or more precisely, sets of motoneurons) is identified with variables in $\mathbf{R}^{\ell}$ so that the phase space of the muscle motoneurons is $Y=\left(\mathbf{R}^{\ell}\right)^{8}$. We also assume that the octahedral group acts on $Y$ by permuting the coordinates, just as that group permutes the vertices of the cube. Next we assume that the differential equations that describe the time evolution of this coupled system of neck motoneurons have octahedral symmetry. Using this symmetry, we can then classify the types of spatiotemporal symmetries that periodic states of such systems may have.

Specifically we find that there are six types of spatiotemporal symmetries for small amplitude periodic solutions that can bifurcate from a $\Gamma$-invariant equilibrium. Each of these symmetry types includes a reasonable pattern of periodic head motion. They are: shaking the head (saying "no" in many cultures), which occurs in two different ways; nodding the head (saying "yes" in those same cultures); a rotating wave in which the head rolls in an approximate horizontal circle; a combination of "yes" and "no," in which the head nods alternately to left and right; and a side-to-side motion with the head rotating to move the nose in the opposite direction (so that the nose always points at a fixed point in the distance).

Organization of the paper. In section 2 we give a brief description of salient features of the physiology of the vestibular system and rederive the octahedral symmetry of the canal-neck projection. We relate the associated network architecture to a graph drawn on a cube and describe a simple caricature of the effects of the eight muscle groups. Section 3 describes the octahedral group in more detail and motivates the choice of action of this group on muscle space. This section also provides an explicit description of the permutation action of the octahedral group on muscle space, lists the relevant subgroups, and classifies the isotropy subgroups - basic data for the application of symmetric dynamics.

The equivariant Hopf theorem is described in section 4, and a discussion of the irreducible representations of the octahedral group, the basic information needed for application of the Hopf theorem, is given. (Proofs, which use character theory, are postponed to the appendix.) Section 5 presents a classification of the possible small amplitude spatiotemporal symmetry patterns for time-periodic motions of the head, determined by the canal-neck projection. We find six distinct (conjugacy classes of) patterns, or 15 distinct patterns (not distinguishing time-reversals or left-right 


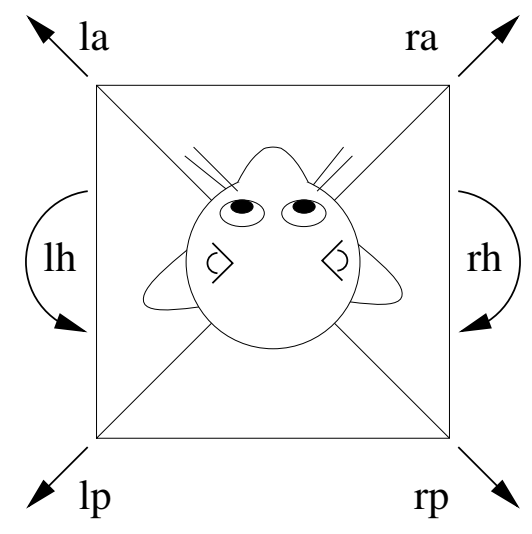

FIG. 1. Location of the three planes relative to the head, and direction of rotational motion to which canals respond. Canals are drawn schematically near the ears.

reflections in physical space). These patterns are interpreted as motions of the head in section 6 , assuming that the muscle groups act according to our caricature. We pay attention to distinctions arising from conjugate states.

We end with a short conclusions section.

2. Symmetries in the disynaptic canal-neck projection. In this section we rederive the symmetries in the disynaptic canal-neck projection discussed by McCollum and Boyle [12], stating the results in terms of a group of permutations acting on the associated network of neurons. In this aspect of the vestibular system there are six canals (three in each ear) that are connected to eight muscle groups in the neck.

The three canals located in each ear are called horizontal $\mathrm{h}$, anterior a, and posterior $\mathrm{p}$. We denote the six canals by $\mathrm{lh}$, la, lp, rh, ra, rp, where l stands for left and $\mathrm{r}$ for right. Neurons associated with canal hairs have a base firing rate. These hairs are arranged so that fluid flow in one direction in the canal increases the firing rate, and fluid flow in the opposite direction decreases that firing rate. Moreover, the canals are paired $(\{\mathrm{lh}, \mathrm{rh}\},\{\mathrm{la}, \mathrm{rp}\},\{\mathrm{lp}, \mathrm{ra}\})$, so that when one member of a pair transmits an elevated signal, then the other member of that pair transmits a reduced one. These pairs are called polarity pairs.

The spatial arrangement of the canals is shown in Figure 1. There are three (approximately) mutually orthogonal planes. One of these planes is horizontal; the other two are vertical, at an angle of $45^{\circ}$ to the plane of left-right symmetry of the head. Each polarity pair consists of two canals that are parallel to one of these planes: one canal in the left ear, one in the right. These two canals are oriented in opposite directions in that plane and detect rotations (actually angular accelerations) of the head about an axis perpendicular to that plane. One member of the polarity pair detects acceleration in one orientation (clockwise or counterclockwise), and the other member detects the opposite orientation, as illustrated by the arrows in Figure 1. The four arrows at the corners represent rotations in the direction "along the arrow and down." For example, ra responds to motion in which the nose and right ear move forward to the left and down, while lp responds to motion in which the nose and right ear move backward to the right and up.

Connections from canals to muscles. Experiments show that each of the six canals can transmit signals to each of the eight muscle groups. The muscles also form 


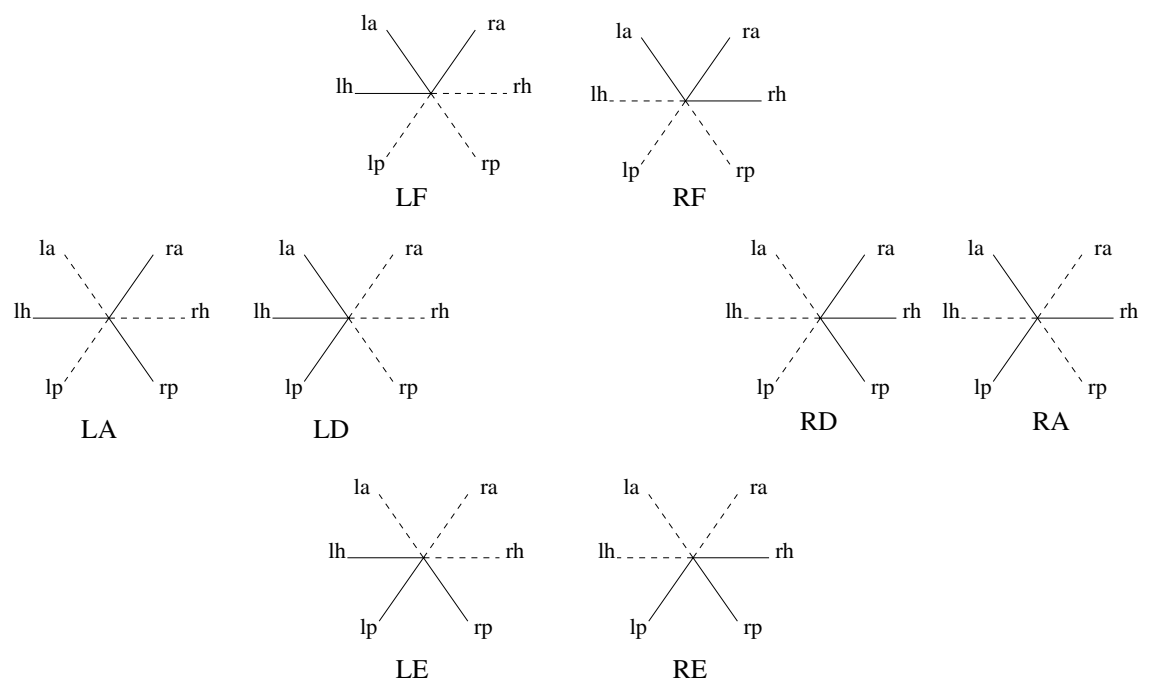

FIG. 2. Innervation patterns corresponding to eight muscle groups. Dashed lines represent excitatory connections and solid lines inhibitory ones.

four polarity pairs; if a canal is activated by the motion of the head, then it sends an excitatory signal to one member of each pair and an inhibitory signal to the other member. Physiological investigations (Wilson and Maeda [16], Shinoda et al. [13, 14, 15]) suggest that each muscle group is excited by a set of three mutually orthogonal canals (that is, one from each polarity pair) and inhibited by the complementary set of canals (the other members of the polarity pairs).

We describe the details of this arrangement, following McCollum and Boyle, who depict the list of signals transmitted to a given muscle group by an "asterisk," (Figure 2). Each asterisk has three solid lines (inhibitory connections) and three dotted lines (excitatory connections), and diametrically opposite lines have opposite polarity. There are eight possible arrangements of this type. Because the asterisks are drawn in 2-dimensional projection, in a conventional orientation with lh between la and lp, there appear to be two kinds of asterisks: two alternating (with excitation and inhibition alternating) and six nonalternating (with three contiguous excitatory canals). We will shortly see that under a suitable action of the octahedral group, all eight asterisks are equivalent.

The eight neck muscles consist of two flexors in the front (LF, RF), two extensors in the back (LE, RE), and four side (shoulder) muscles. The side muscles are alternating (LA, RA) or directed (LD, RD). McCollum and Boyle [12] discuss the innervation patterns between canal neurons and muscle motoneurons - how the six canal neurons connect to the eight muscle motoneurons, and whether the connection occurs via an excitatory synapse or an inhibitory one. The pattern of connections to each muscle is specified by Figure 2. Each asterisk in Figure 2 is a list of the connections from all six canals to one muscle group, and the type of signal that is transmitted along each connection. Observe that the muscle groups also partition into four polarity pairs:

$$
\{L A, R A\}, \quad\{L F, R E\}, \quad\{L E, R F\}, \quad\{L D, R D\} .
$$

If one muscle in a polarity pair has an excitatory connection from a canal, then the other muscle in that polarity pair has an inhibitory connection from that canal. 


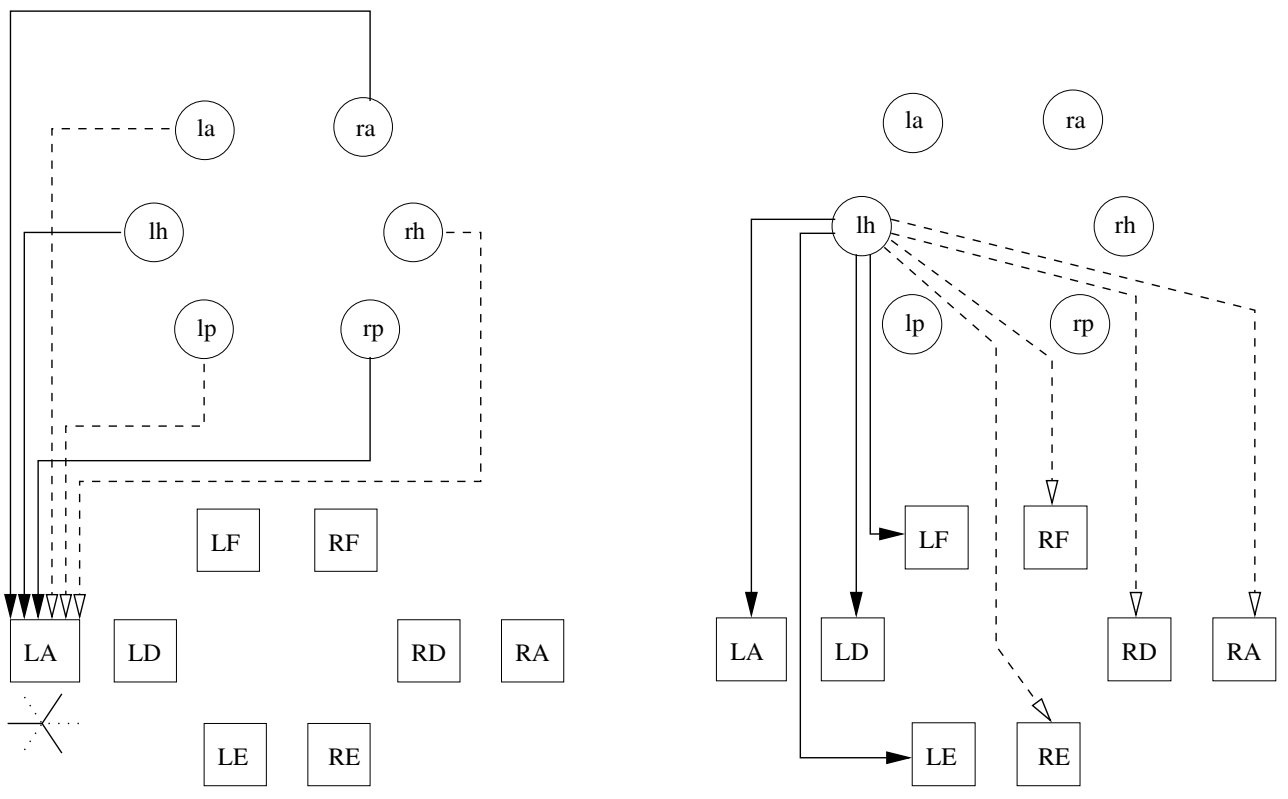

FIG. 3. Schematic of connections from vestibular nerve afferent to neck motoneuron. Solid line shows inhibitory synapse, dotted line shows excitatory synapse. Left: Connections to a given neck motoneuron, here LA. Right: Connections from a given vestibular nerve afferent, here lh.

It is useful to display the same information in two other ways. McCollum and Boyle [12] consider only the disynaptic pathway from the six vestibular nerve afferents ("canal nerves") to the eight neck motoneurons (by way of the corresponding vestibulospinal neurons). They remark that almost always "the motoneurons of each tested muscle responded to stimulation of all six canal nerves." The responses were classified as either inhibitory or excitatory, as indicated by solid or dotted lines for the relevant arm of the asterisk. This description makes it clear that their Figure 3 (and our Figure 2) is a diagram determining these connections.

We make the connection pattern explicit. Figure 3(left) shows connections to a given neck motoneuron, here LA. The associated asterisk is drawn, and the six connections correspond to the six arms. Figure 3(right) shows connections from a given vestibular nerve afferent, here $\mathrm{lh}$. These connections correspond to the eight $\mathrm{lh}$ arms in the different asterisks, and connect to the corresponding neck motoneurons.

We do not attempt to draw the complete network since it would contain 48 lines, 24 solid and 24 dotted, and it would be too complicated to convey useful information. However, it is convenient to employ a geometric image in which the canals are identified with the six faces of a cube, and the muscles with the eight vertices. We will describe the network connectivity using the cube.

Octahedral symmetry of canals and muscles. The cube arises naturally from the results of McCollum and Boyle [12], identifying the symmetry group of the canal-neck projection as the 48-element octahedral group. To understand their observation, we identify the canals with faces of a cube, so that polarity pairs of canals are identified with pairs of opposite faces. Up to symmetry there is only one way to make this identification.

To identify the muscles, we observe that every vertex of the cube is in the inter- 


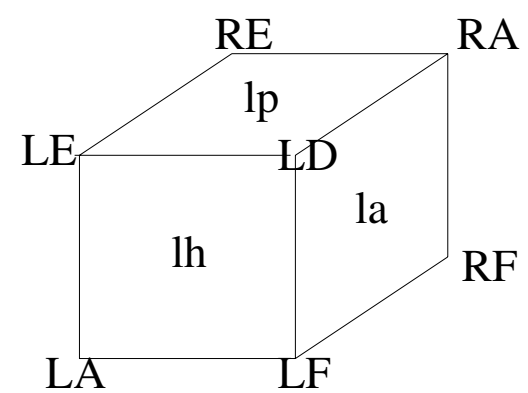

FIG. 4. Identification of polarity pairs and muscle groups to the cube.

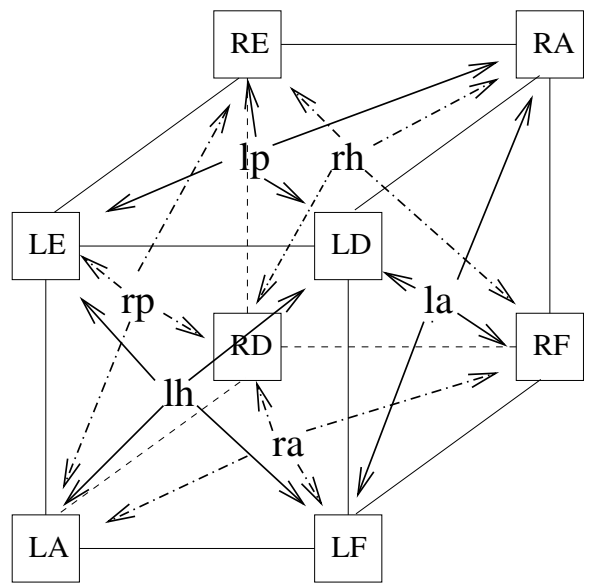

FIG. 5. Schematic of inhibitory connections from canals to muscles drawn on the cube. Solid lines show connections on "visible" faces, and dot/dashed lines show connections on "hidden" faces. Canals are at centers of faces, muscles at vertices. Connections run to each vertex from the three adjacent faces. The octahedral symmetry of the network is obvious geometrically.

section of exactly three faces. We identify a given vertex with that muscle that has inhibitory connections from canals corresponding to the three faces adjacent to that vertex. For example, there is a unique vertex that is in the intersection of the three faces corresponding to the left canals lh, lp, la (see Figure 4). We identify this vertex with the left direct muscle LD in Figure 2, since that muscle responds to inhibitory signals from the three left canals.

In Figure 5 we show the 24 inhibitory connections on the cube diagram. The complementary set of connections from canal neurons to muscle motoneurons consists of excitatory connections but is omitted for clarity. The octahedral symmetry of the network is apparent in this figure. The elements of the octahedral group act on the full network by permuting canals, permuting muscles, and permuting the corresponding connections.

Muscle group action: A caricature. What effect do the eight muscle groups have on the head? For purposes of interpretation, we adopt a caricature of the anatomy of the muscle groups, illustrated in Figure 6. Here we assume that the principal effect of a muscle group being activated is to pull the head in the indicated direction. Six muscle groups LF, LD, LE, RF, RD, RE effectively form a "hexagon," and their effect is to tilt the head in various directions. The other two, LA and RA, 


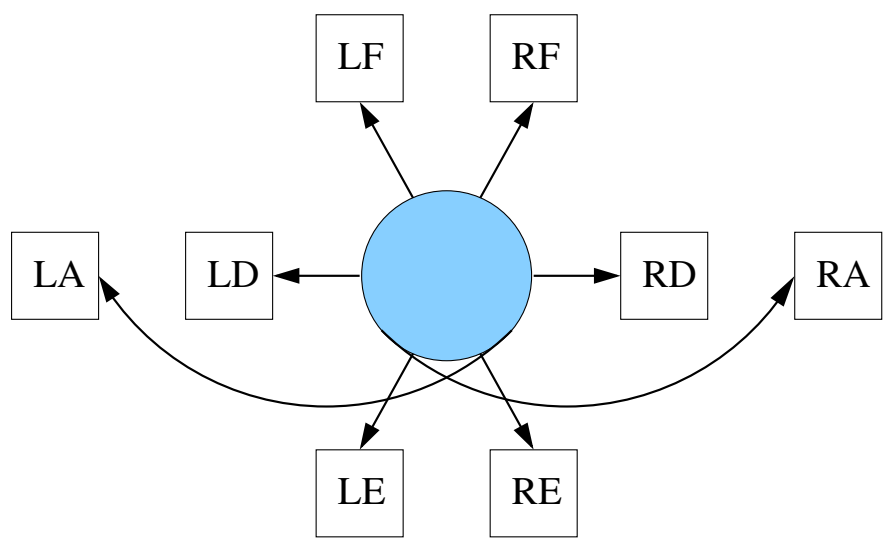

FIG. 6. Caricature of effect of activation of muscle groups.

rotate the head about the vertical axis (as sensed by the horizontal canals lh, rh). There is some redundancy here: the hexagon includes three pairs of muscle groups, but the three associated directions are linearly dependent. However, the use of six muscles makes the head position more stable, so there may be physiological reasons for this redundancy. McCollum and Boyle [12] call this hexagon the "central dial." This caricature exhibits the four pairs of opposing muscles (LD, RD), (LE, RF), (LF, RE), (LA, RA), which are just the four polarity pairs.

We stress that this picture of the anatomy is a caricature. At this stage we make no attempt to formulate a more realistic model of the physiology and the mechanics of head movement. However, further detail of this kind could be developed without changing the classification of possible symmetry types of time-periodic motion. What would change would be the fine detail of the corresponding head motions and the precise manner in which each muscle group contributes to that motion.

3. The octahedral group and its actions. We now discuss mathematical features of the octahedral group and various actions of that group that occur in this analysis. In particular, we introduce variables that model the state of the eight muscle groups and discuss how the octahedral group acts on those variables.

The geometry of Figure 5, together with the corresponding figure for excitatory connections (which has the same symmetry), shows that the network of neurons forming the canal-neck projection has octahedral symmetry, where now the octahedral group acts by permuting the eight muscle groups, the six canal neurons, and the connections between them. These permutation actions are distinct from, but induced naturally by, the "standard" action as isometries of $\mathbf{R}^{3}$ that preserve the cube.

Suppose we fix the cube so that it is centered at the origin. Then the symmetries of the cube have the form $R$ or $-R$, where $R$ is a rotation. It follows that the octahedral group is the direct sum of the group $\mathbb{O}$ of rotation symmetries of the cube and the two-element group $\mathbf{Z}_{2}^{c}$ generated by the inversion $-I$. That is, the octahedral group is $\mathbb{O} \oplus \mathbf{Z}_{2}^{c}$. The " $c$ " in the notation $\mathbf{Z}_{2}^{c}$ indicates that this group is the center of the octahedral group.

We are modeling the canal-neck projection by a network of interconnecting neurons, following Figures 3 and 5 . This network has symmetry group $\mathbb{O} \oplus \mathbf{Z}_{2}^{c}$, which acts on the network by permuting the set of cells and the set of arrows. This permutation action preserves the type of the cell (canal neuron, shown as a circle, or muscle 
motoneuron, shown as a square), and it preserves the type of arrow (inhibitory or excitatory).

Phase space for muscles. This permutation action can be transferred to the dynamical variables representing the states of the cells, that is, the phase space of the network. We now describe the effect of this action on the 8 muscle cells. In order to do this we order the eight vertices as in (3.1). The ordering is shown on the cube in Figure 7 .

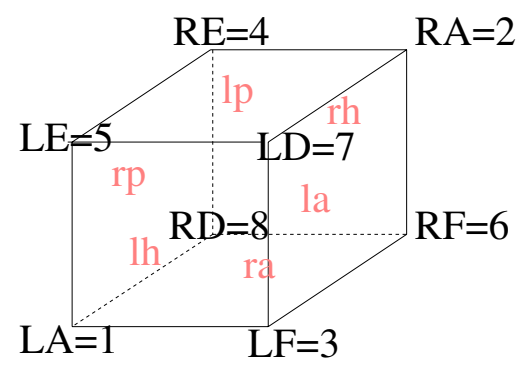

Fig. 7. Ordering of vertices.

The simplest model for a state of the eight muscle motoneurons is a point in $\mathbf{R}^{8}$, with coordinates

$$
\left(y_{\mathrm{LA}}, y_{\mathrm{RA}}, y_{\mathrm{LF}}, y_{\mathrm{RE}}, y_{\mathrm{LE}}, y_{\mathrm{RF}}, y_{\mathrm{LD}}, y_{\mathrm{RD}}\right)
$$

Each element of $\mathbb{O} \oplus \mathbf{Z}_{2}^{c}$ permutes the eight subscripts LA, RA, LF, RE, LE, RF, $\mathrm{LD}, \mathrm{RD}$ according to the associated transformation of the vertices of the cube in Figure 7. The overall phase space for any system of ODEs representing the dynamics of the network, consistent with the $\mathbb{O} \oplus \mathbf{Z}_{2}^{c}$ symmetry, is therefore equivariant for the permutation action of $\mathbb{O} \oplus \mathbf{Z}_{2}^{c}$ on the space $\mathbf{R}^{8}$.

Our goal is to determine the spatiotemporal symmetries of small amplitude periodic solutions that can be obtained from a synchronous equilibrium by Hopf bifurcation. An important step in this analysis is the computation of the irreducible representations of the symmetry group $\mathbb{O} \oplus \mathbf{Z}_{2}^{c}$ on $\left(\mathbf{R}^{\ell}\right)^{8}$. However, up to isomorphism the answer for general $\ell$ is identical to the case when $\ell=1$, for the following reason. Because the group acts by permutations (see the next subsection), the action on $\left(\mathbf{R}^{\ell}\right)^{8}$ consists of $\ell$ isomorphic copies of the action on $\mathbf{R}^{8}$. So the isomorphism types of the irreducible components are the same for all $\ell$. However, their multiplicities depend on $\ell$. We return to this point in section 4 .

The action of $\mathbb{O} \oplus \mathbf{Z}_{2}^{c}$ is determined by that of $\mathbb{O}$ and that of $\mathbf{Z}_{2}^{c}$. A crucial feature of the "cube" structure is that the action of $-I$ preserves polarity pairs $\{\mathrm{LA}, \mathrm{RA}\}$, $\{\mathrm{LF}, \mathrm{RE}\},\{\mathrm{LE}, \mathrm{RF}\}$, and $\{\mathrm{LD}, \mathrm{RD}\}$, because they label pairs of opposite vertices. Their entries are interchanged by $-I$. That is, $-I$ acts as the permutation

$$
\text { (1 2)(3 4)(5 6)(7 8). }
$$

Permutation action of $\mathbb{O}$ on muscle space. It remains to analyze the action of $\mathbb{O}$. We begin the discussion of the action of $\mathbb{O}$ on $\mathbf{R}^{8}$ by describing how it acts by rotations on $\mathbf{R}^{3}$ in the "cube" picture. There are three types of rotation: rotations about axes connecting centers of opposite faces, rotations about axes connecting midpoints of opposite edges, and rotations about axes containing opposite vertices. There 
TABLE 1

Permutation actions on $\mathbf{R}^{8}$ of rotations in $\mathbb{O}$.

\begin{tabular}{|c|c|}
\hline$I$ & $I$ \\
\hline$V_{1}$ & $(385)(476)$ \\
\hline$V_{2}$ & $(358)(467)$ \\
\hline$V_{3}$ & $(176)(285)$ \\
\hline$V_{4}$ & $(167)(258)$ \\
\hline$V_{5}$ & $\left(\begin{array}{l}14 \\
4\end{array}\right)(238)$ \\
\hline$V_{6}$ & $(174)(283)$ \\
\hline$V_{7}$ & $(146)(235)$ \\
\hline$V_{8}$ & $(164)(253)$ \\
\hline$A_{1}$ & $(17)(28)(35)(46)$ \\
\hline$A_{2}$ & $(14)(23)(58)(67)$ \\
\hline$A_{3}$ & $(16)(25)(38)(47)$ \\
\hline
\end{tabular}

\begin{tabular}{|c|c|}
\hline$F_{1}$ & $(1573)(2684)$ \\
\hline$F_{2}$ & $(1375)(2486)$ \\
\hline$F_{3}$ & $(1548)(2637)$ \\
\hline$F_{4}$ & $(1845)(2736)$ \\
\hline$F_{5}$ & $(1863)(2754)$ \\
\hline$F_{6}$ & $(1368)(2457)$ \\
\hline$E_{13}$ & $(12)(37)(48)(56)$ \\
\hline$E_{14}$ & $(15)(26)(34)(78)$ \\
\hline$E_{15}$ & $(12)(34)(57)(68)$ \\
\hline$E_{16}$ & $(13)(24)(56)(78)$ \\
\hline$E_{35}$ & $(18)(27)(34)(56)$ \\
\hline$E_{45}$ & $(12)(36)(45)(78)$ \\
\hline
\end{tabular}

TABLE 2

The 10 nonidentity subgroups of $\mathbb{O}$ up to conjugacy, with generators.

\begin{tabular}{|c|c|l|c|}
\hline Subgroup & Order & Generators & Normalizer \\
\hline $\mathbf{Z}_{2}^{A}$ & 2 & $A_{3}$ & $\mathbf{D}_{4}$ \\
\hline $\mathbf{Z}_{2}^{E}$ & 2 & $E_{16}$ & $\mathbf{D}_{2}^{E}$ \\
\hline $\mathbf{Z}_{3}$ & 3 & $V_{1}$ & $\mathbf{S}_{3}$ \\
\hline $\mathbf{D}_{2}^{A}$ & 4 & $A_{1}, A_{3}$ & $\mathbb{O}$ \\
\hline $\mathbf{D}_{2}^{E}$ & 4 & $E_{15}, E_{16}$ & $\mathbf{D}_{4}$ \\
\hline \hline Subgroup & Order & Generators & Normalizer \\
\hline $\mathbf{Z}_{4}$ & 4 & $F_{3}$ & $\mathbf{D}_{4}$ \\
\hline $\mathbf{S}_{3}$ & 6 & $E_{15}, V_{1}$ & $\mathbf{S}_{3}$ \\
\hline $\mathbf{D}_{4}$ & 8 & $A_{3}, F_{3}$ & $\mathbf{D}_{4}$ \\
\hline $\mathbb{T}$ & 12 & $A_{3}, V_{4}$ & $\mathbb{O}$ \\
\hline $\mathbb{O}$ & 24 & $V_{4}, F_{5}$ & $\mathbb{O}$ \\
\hline
\end{tabular}

are nine rotations corresponding to faces, since there are three pairs of faces and each pair determines three nonidentity rotations. There are six rotations corresponding to edges, since there are six pairs of edges and each pair determines just one nonidentity rotation. There are eight rotations corresponding to vertices, since there are four pairs of vertices and each pair determines two nonidentity rotations.

Denote by $V_{j}$ the clockwise rotation of $120^{\circ}$ about the axis through vertex $j$ for $j=1, \ldots, 8$ ("clockwise" when viewed with vertex $j$ nearest to the eye). Note that $V_{1}^{2}=V_{2}, V_{3}^{2}=V_{4}, V_{5}^{2}=V_{6}, V_{1}^{7}=V_{8}$. Denote by $F_{j}$ the clockwise rotation of $90^{\circ}$ about the axis perpendicular to face $j$ for $j=1, \ldots, 6$. Note that $F_{1}^{3}=F_{2}, F_{3}^{3}=F_{4}$, $F_{5}^{3}=F_{6}$. Let $A_{i}=F_{2 i-1}^{2}$ for $i=1,2,3$. Then the $F_{j}$ and the $A_{i}$ are the nine rotations about axes connecting midpoints of opposite faces. Finally, note that each edge is uniquely the intersection of two faces. Denote by $E_{i j}$ the rotation by $180^{\circ}$ about the edge in the intersection of faces $i$ and $j$, where $i<j$. There are six possibilities.

In Table 1 we list the 24 rotations and their permutation actions on $\mathbf{R}^{8}$. The entries can be read off easily from Figure 7 .

Subgroups of $\mathbb{O}$. We use the following notation for groups: $\mathbf{Z}_{k}$ is the cyclic group of order $k, \mathbf{D}_{k}$ is the dihedral group of order $k, \mathbf{S}_{k}$ is the symmetric group of degree $k$, and $\mathbb{T}$ is the tetrahedral group. This is the unique subgroup of $\mathbb{O}$ that has order 12, and it fixes a tetrahedron inscribed in the cube. Table 2 lists the 11 conjugacy classes of subgroups of $\mathbb{O}$. This calculation was done using the algebra program GAP. 
4. Types of Hopf bifurcation. Hopf bifurcation is the tool for finding small amplitude periodic states near an equilibrium. Equivariant Hopf theory [8, 9] states that there is a different type of Hopf bifurcation from a group-invariant equilibrium for each irreducible representation of the group. The equivariant Hopf theorem helps classify the types of spatiotemporal symmetries of periodic states that emanate from a given Hopf bifurcation. We apply this theory in the case of $\mathbb{O} \oplus \mathbf{Z}_{2}^{c}$ acting on muscle space $\left(\mathbf{R}^{\ell}\right)^{8}$, where the equilibrium is $\mathbb{O} \oplus \mathbf{Z}_{2}^{c}$-invariant. At such an equilibrium opposing muscles act with equal strength, so that the head is fixed and upright.

As noted previously, the types of irreducible representation of $\mathbb{O} \oplus \mathbf{Z}_{2}^{c}$ acting on $\left(\mathbf{R}^{\ell}\right)^{8}$ are identical with those of $\mathbb{O} \oplus \mathbf{Z}_{2}^{c}$ acting on $\mathbf{R}^{8}$. So the first step is to find the irreducible representations of $\mathbb{O} \oplus \mathbf{Z}_{2}^{c}$ acting on $\mathbf{R}^{8}$. We will show that there are four different irreducible representations, only two of which can lead to periodic states corresponding to nontrivial head motions. One of the associated Hopf bifurcations is simple to analyze, and the other was analyzed previously by Ashwin and Podvigina [1].

Decomposition of $\mathbf{R}^{8}$ into "push-pull" and "pull-pull" subspaces. The irreducible representations are intimately associated with the action of the inversion $-I$, which plays a key role because it swaps the members of each pair of opposing muscle motoneurons.

We can decompose $\mathbf{R}^{8}=Y^{+} \oplus Y^{-}$into two 4-dimensional $\mathbb{O} \oplus \mathbf{Z}_{2}^{c}$-invariant subspaces, so that $-I$ acts trivially on one subspace and changes sign on the other. To do so, define

$$
Y^{ \pm}=\left\{y \in \mathbf{R}^{8}: y_{\mathrm{LA}}= \pm y_{\mathrm{RA}}, y_{\mathrm{LF}}= \pm y_{\mathrm{RE}}, y_{\mathrm{LE}}= \pm y_{\mathrm{RF}}, y_{\mathrm{LD}}= \pm y_{\mathrm{RD}}\right\} .
$$

Note that the coordinates corresponding to opposing muscle pairs in $Y^{+}$are equal, and the coordinates corresponding to opposing muscle pairs in $Y^{-}$are equal in magnitude but opposite in sign.

As noted previously, three polarity pairs of muscles (the central dial) pull the head in opposite directions, and the muscles of the fourth pair (the alternating muscles) twist the head in opposite directions. In states in $Y^{+}$polarity pairs of muscles act as pull-pull pairs, whereas in states in $Y^{-}$these polarity pairs act as push-pull pairs. In fact, all muscles must be under tension; thus push-pull pairs really operate with one muscle group pulling harder than usual while the other pulls less hard. Phenomenologically, we can identify the difference between the tensions of two muscles in a polarity pair with the deviation of the tension (of either muscle, subject to sign) from the rest tension in which the head remains upright.

Next we observe that Hopf bifurcation corresponding to an irreducible representation in $Y^{+}$can only lead to periodic states in which the head is immobile. The reason is simple: $Y^{+}=\operatorname{Fix}(-I)$, which is flow-invariant. Thus, in the nonlinear theory, any periodic state emanating from such a bifurcation must itself be fixed by $-I$; consequently, the opposing muscles in each polarity pair are always pulling with the same strength, creating a net motion of zero. As well as being inefficient, this space of motions has no visible effect on the head. In contrast, on the space $Y^{-}$, opposing pairs of muscles cooperate to move the head in exactly the same manner, so the muscle actions reinforce each other.

In fact, neither subspace $Y^{+}$or $Y^{-}$is irreducible; each subspace decomposes into a 1-dimensional and a 3-dimensional irreducible representation. The previous remark implies that we need focus only on the subspace $Y^{-} \cong \mathbf{R}^{4}$.

Decomposition of $\boldsymbol{Y}^{-}$into irreducible subspaces. As we have seen, the inversion $-I$ interchanges the muscles in each polarity pair, and the states in $Y^{-}$are 
TABLE 3

Action of elements in $\mathbb{O}$ on muscle "push-pull" polarity pair space $Y^{-}$.

\begin{tabular}{|c|c|}
\hline$\gamma$ & Action on $\gamma$ on $Y^{-}$ \\
\hline$I$ & $\left(y_{1}, y_{3}, y_{5}, y_{7}\right)$ \\
\hline$V_{1}$ & $\left(y_{1}, y_{5},-y_{7},-y_{3}\right)$ \\
$V_{2}$ & $\left(y_{1},-y_{7}, y_{3},-y_{5}\right)$ \\
$V_{3}$ & $\left(-y_{5}, y_{3},-y_{7}, y_{1}\right)$ \\
$V_{4}$ & $\left(y_{7}, y_{3},-y_{1},-y_{5}\right)$ \\
$V_{5}$ & $\left(y_{7},-y_{1}, y_{5},-y_{3}\right)$ \\
$V_{6}$ & $\left(-y_{3},-y_{7}, y_{5}, y_{1}\right)$ \\
$V_{7}$ & $\left(-y_{5},-y_{1}, y_{3}, y_{7}\right)$ \\
$V_{8}$ & $\left(-y_{3}, y_{5},-y_{1}, y_{7}\right)$ \\
\hline$A_{1}$ & $\left(y_{7}, y_{5}, y_{3}, y_{1}\right)$ \\
$A_{2}$ & $\left(-y_{3},-y_{1},-y_{7},-y_{5}\right)$ \\
$A_{3}$ & $\left(-y_{5},-y_{7},-y_{1},-y_{3}\right)$ \\
\hline
\end{tabular}

\begin{tabular}{|c|c|}
\hline$\gamma$ & Action on $\gamma$ on $Y^{-}$ \\
\hline$F_{1}$ & $\left(y_{3}, y_{7}, y_{1}, y_{5}\right)$ \\
$F_{2}$ & $\left(y_{5}, y_{1}, y_{7}, y_{3}\right)$ \\
$F_{3}$ & $\left(-y_{7},-y_{5}, y_{1}, y_{3}\right)$ \\
$F_{4}$ & $\left(y_{5}, y_{7},-y_{3},-y_{1}\right)$ \\
$F_{5}$ & $\left(y_{3},-y_{5}, y_{7},-y_{1}\right)$ \\
$F_{6}$ & $\left(-y_{7}, y_{1},-y_{3}, y_{5}\right)$ \\
\hline$E_{13}$ & $\left(-y_{1}, y_{7},-y_{5}, y_{3}\right)$ \\
$E_{14}$ & $\left(y_{5},-y_{3}, y_{1},-y_{7}\right)$ \\
$E_{15}$ & $\left(-y_{1},-y_{3}, y_{7}, y_{5}\right)$ \\
$E_{16}$ & $\left(y_{3}, y_{1},-y_{5},-y_{7}\right)$ \\
$E_{35}$ & $\left(-y_{7},-y_{3},-y_{5},-y_{1}\right)$ \\
$E_{45}$ & $\left(-y_{1},-y_{5},-y_{3},-y_{7}\right)$ \\
\hline
\end{tabular}

ones of the form

$$
\left(y_{\mathrm{LA}},-y_{\mathrm{LA}}, y_{\mathrm{LF}},-y_{\mathrm{LF}}, y_{\mathrm{LE}},-y_{\mathrm{LE}}, y_{\mathrm{LD}},-y_{\mathrm{LD}}\right)
$$

that is, we can parametrize $Y^{-}$by the strengths of the four left muscle groups, which correspond to the muscle groups numbered $1,3,5,7$. Thus we can rewrite (4.1) as

$$
\left(y_{1},-y_{1}, y_{3},-y_{3}, y_{5},-y_{5}, y_{7},-y_{7}\right)
$$

which we parametrize by $\left(y_{1}, y_{3}, y_{5}, y_{7}\right)$.

On $Y^{-}$the action of $\mathbb{O} \oplus \mathbf{Z}_{2}^{c}$ can now be written using signed permutations, since this action preserves polarity pairs and introduces a minus sign when members of a polarity pair are swapped. In particular, we can identify the action of $-I$ on $Y^{-}$with the signed permutation $\left(-y_{1},-y_{3},-y_{5},-y_{7}\right)$; that is, $-I$ acts by multiplication by -1 on $Y^{-}$, as expected. The signed permutation action of $\mathbb{O}$ on $Y^{-}$is given in Table 3.

The subspace $Y^{-}$contains the 1-dimensional (hence irreducible) subspace

$$
Y_{0}^{-}=\mathbf{R}\{(1,-1,-1,1,-1,1,1,-1)\}
$$

upon which the elements $A_{3}$ and $V_{4}$, the generators of the tetrahedral group, act trivially. In addition, $(\mathbb{O} \backslash \mathbb{T},-I)$ acts trivially, since both $\mathbb{O} \backslash \mathbb{T}$ and $-I$ act as multiplication by -1 .

Let $Y_{1}^{-}$be the 3-dimensional invariant complement of $Y_{0}^{-}$in $Y^{-}$; so $Y^{-}=Y_{0}^{-} \oplus$ $Y_{1}^{-}$. It can be shown that $Y_{1}^{-}$is irreducible, and the action of $\mathbb{O}$ on $Y_{1}^{-}$is isomorphic to the standard action of the cube on $\mathbf{R}^{3}$. We do this using character theory in the appendix.

Recall that for modeling purposes we assume that the muscle state space $Y^{-}$ consists of $\ell$ variables for each polarity pair of muscles. Thus $Y^{-} \cong\left(\mathbf{R}^{\ell}\right)^{4}$. As noted previously, the minimal phase space for any of our models occurs when $\ell=1$. Although the analysis of possible spatiotemporal patterns reduces to the case $\ell=1$, when we come to consider Hopf bifurcation, it turns out that we must require $\ell \geq$ 2. (Reason: equivariant Hopf bifurcation requires certain representations to appear twice, namely, the absolutely irreducible ones, and that multiplicity occurs only when $\ell \geq 2$. See $[8,9]$.) Since all neurons, and in particular muscle motoneurons, have high-dimensional internal dynamics, this condition poses no difficulties. 
5. Symmetry types of periodic state. At a $\Gamma$-invariant equilibrium for a $\Gamma$ equivariant system of ODEs, the equivariant Hopf theorem [8, 9] states (under several genericity hypotheses) that there exists a branch of small amplitude periodic states corresponding to every $\mathbf{C}$-axial subgroup of $\Gamma \times \mathbf{S}^{1}$ acting on the center subspace at that equilibrium. Moreover, these periodic states have spatiotemporal symmetries given by the $\mathbf{C}$-axial subgroup. A subgroup of $\Gamma \times \mathbf{S}^{1}$ is $\mathbf{C}$-axial if it is an isotropy subgroup, and its fixed-point subspace, within the eigenspace corresponding to the purely imaginary eigenvalues, has dimension 2 .

A complete discussion of equivariant Hopf theory is beyond the scope of this paper; details can be found in $[8,9]$. To simplify the remarks we make here, we assume that all periodic solutions have period 1 . Then $\mathbf{S}^{1}$, the group of phase shift symmetries, is parameterized from 0 to 1 . We now recall two general points from Hopf theory. First, the phase shift by $\frac{1}{2}$ acts as multiplication by -1 on the center subspace. Second, the kernel of the action of $\Gamma \times \mathbf{S}^{1}$ on the center subspace is contained in every C-axial subgroup.

In the case at hand, we saw that $-I$ acts as multiplication by -1 on $Y^{-}$. Thus the element $\left(-I, \frac{1}{2}\right)$ in $\mathbf{Z}_{2}^{c} \times \mathbf{S}^{1}$ acts trivially in any Hopf bifurcation with center subspace in $Y^{-}$. It follows that every periodic state emanating from such a bifurcation has the property that interchanging polarity pairs is the same as making a half period phase shift. That is,

$$
\begin{array}{ll}
y_{2}\left(t+\frac{1}{2}\right)=y_{1}(t), & y_{4}\left(t+\frac{1}{2}\right)=y_{3}(t), \\
y_{6}\left(t+\frac{1}{2}\right)=y_{5}(t), & y_{8}\left(t+\frac{1}{2}\right)=y_{7}(t) .
\end{array}
$$

Dividing by the subgroup $\mathbf{Z}_{2}\left(-I, \frac{1}{2}\right)$ leads to the standard action of $\mathbb{O} \times \mathbf{S}^{1}$ on the center subspace. To see this, consider the epimorphism $\varphi:\left(\mathbb{O} \oplus \mathbf{Z}_{2}^{c}\right) \times \mathbf{S}^{1} \rightarrow \mathbb{O} \times \mathbf{S}^{1}$ defined by

$$
\varphi(\gamma, I, \theta)=(\gamma, \theta) \quad \text { and } \quad \varphi(\gamma,-I, \theta)=\left(\gamma, \theta+\frac{1}{2}\right) .
$$

The kernel of $\varphi$ is $\mathbf{Z}_{2}\left(-I, \frac{1}{2}\right)$, and the quotient group is $\mathbb{O} \times \mathbf{S}^{1}$ with its standard action on $Y^{-}$, since $\varphi(\gamma, I, \theta)=(\gamma, \theta)$. It follows that to classify the relevant types of periodic solutions, we need analyze only those periodic solutions that occur in Hopf bifurcations associated to $\mathbb{O}$ acting on $Y^{-}$and then add in the constraints (5.1), if needed.

Using the decomposition of phase space into $Y^{+}$and $Y^{-}$components, we can write any periodic state in the form $y(t)=y^{+}(t)+y^{-}(t)$. When we come to interpret the motions associated with the periodic states, factoring out the $Y^{+}$component will not change these motions in any important manner since, as discussed previously, $y^{+}(t)$ by itself leaves the head immobile. Moreover, near these Hopf bifurcations the $Y^{+}$components will be small compared to the $Y^{-}$components. More precisely, suppose that a Hopf bifurcation supported in $Y^{-}$leads to a periodic state of amplitude $\varepsilon$. Then the theory implies that generically $y^{-}(t)$ will be of order $\varepsilon$, while $y^{+}(t)$ will be of order $\varepsilon^{2}$. Finally, coupling (5.1) with the definitions of $Y^{-}$and $Y^{+}$leads to the conclusion that $y^{+}(t)$ oscillates with twice the frequency of $y^{-}(t)$ and that

$$
\begin{array}{ll}
y_{1}^{-}\left(t+\frac{1}{2}\right)=-y_{1}^{-}(t), & y_{3}^{-}\left(t+\frac{1}{2}\right)=-y_{3}^{-}(t), \\
y_{5}^{-}\left(t+\frac{1}{2}\right)=-y_{5}^{-}(t), & y_{7}^{-}\left(t+\frac{1}{2}\right)=-y_{7}^{-}(t) .
\end{array}
$$

In short, when discussing small amplitude periodic solutions of the nonlinear ODEs on muscle phase space, the system can effectively be reduced to an $\mathbb{O}$-equivariant 
system of ODEs on the reduced phase space $Y^{-}$whose periodic solutions also satisfy (5.2). It is this reduced system that we study for the remainder of this paper.

Spatiotemporal symmetries defined by $\boldsymbol{H}$ and $\boldsymbol{K}$. In $\Gamma$-equivariant systems we can associate two subgroups $H$ and $K$ of $\Gamma$ to each periodic state $y(t)$. Elements of the subgroup $K$ fix the periodic trajectory pointwise, whereas elements of the subgroup $H$ fix the periodic trajectory setwise. Uniqueness of solutions with a given initial condition implies that each element of $H$ couples with a phase shift to fix the periodic state.

When $\ell \geq 2$, periodic states can have spatiotemporal symmetry group pairs $(H, K)$ only if $H / K$ is cyclic and $K$ is an isotropy subgroup [9]. We describe the symmetries associated with periodic states obtained by Hopf bifurcation in terms of these $(H, K)$ pairs.

Hopf bifurcation in $\boldsymbol{Y}^{-}$. Next we classify the types of periodic state that can arise as a small amplitude motion near the steady state (in which there is no head motion). Such states can be found using the equivariant Hopf theorem [8, 9]. This theorem states that there is a possible Hopf bifurcation corresponding to each irreducible representation of $\mathbb{O}$ acting on phase space. Now, the decomposition of $Y^{-}$ into irreducibles can be viewed as a decomposition $\mathbf{R}^{4}=W_{0} \oplus W_{1}$, where

$$
W_{0}=\mathbf{R}\{(1,-1,-1,1)\} \quad \text { and } \quad W_{1}=\mathbf{R}\{(1,1,1,1),(1,1,-1,-1),(1,-1,1,-1)\} .
$$

Here $W_{0}$ corresponds to $Y_{0}^{-}$, and $W_{1}$ corresponds to $Y_{1}^{-}$. Both are irreducible. The kernel of the action of $\mathbb{O}$ on $W_{0}$ is $\mathbb{T}$; the representation on $W_{1}$ is the standard 3dimensional irreducible representation, in which $\mathbb{O}$ acts as isometries that preserve the cube.

Hopf bifurcation via $W_{0}$ leads to periodic states with $H=\mathbb{O}$ and $K=\mathbb{T}$. Ashwin and Podvigina [1] classify the periodic states that arise from the standard irreducible representation of $\mathbb{O}$. This is the difficult case for Hopf bifurcation. There are five types of periodic state, whose $(H, K)$ pairs are $\left(\mathbf{D}_{4}, \mathbf{Z}_{4}\right),\left(\mathbf{D}_{2}^{E}, \mathbf{Z}_{2}^{E}\right),\left(\mathbf{Z}_{4}, \mathbf{1}\right),\left(\mathbf{S}_{3}, \mathbf{Z}_{3}\right)$, and $\left(\mathbf{Z}_{3}, \mathbf{1}\right)$. Table 4 lists these pairs, together with associated information.

We sketch the derivation of the "muscle oscillation" column of this table. Consider the pair $(H, K)=(\mathbb{O}, \mathbb{T})$ in the first row. Here $\mathbb{T}$ fixes the state of each muscle group at each time. By Table 2 , the group $\mathbb{T}$ is generated by $A_{3}$ and $V_{4}$. Therefore, by Table 3 , any state $y(t)$ with the symmetry pair $(\mathbb{O}, \mathbb{T})$ must satisfy

$$
y_{1}(t) \equiv-y_{5}(t), \quad y_{3}(t) \equiv-y_{7}(t), \quad y_{1}(t) \equiv y_{7}(t),
$$

so that

$$
y(t)=(u(t),-u(t),-u(t), u(t))
$$

for a time-periodic function $u$. The quotient $H / K$ is isomorphic to $\mathbf{Z}_{2}$ and is generated (modulo $K$ ) by the element $\left(F_{5}, \frac{1}{2}\right) \in \mathbb{O} \times \mathbf{S}^{1}$. This imposes the same condition $u\left(t+\frac{1}{2}\right)=-u(t)$ that was previously noted using the symmetry $\left(-I, \frac{1}{2}\right)$.

For a more complicated example, consider the pair $(H, K)=\left(\mathbf{Z}_{3}, \mathbf{1}\right)$. Since $K$ is trivial, no components of $y(t)$ are forced to be synchronous. The subgroup $\mathbf{Z}_{3}$ is generated by $V_{1}$, whose action on $\mathbf{R}^{4}$ fixes $y_{1}$ and cycles $\left(y_{3}, y_{5},-y_{7}\right)$. The only possible pattern of phase shifts here is $\left(0, \frac{1}{3} \delta, \frac{2}{3} \delta\right)$, where $\delta= \pm 1$. So

$$
\left(y_{3}(t), y_{5}(t), y_{7}(t)\right)=\left(u(t), u\left(t+\frac{1}{3} \delta\right),-u\left(t+\frac{2}{3} \delta\right)\right)
$$


TABLE 4

Conjugacy classes of Hopf-type states, and the associated patterns of muscle activation, where $\delta= \pm 1, u\left(t+\frac{1}{2}\right)=-u(t), z\left(t+\frac{1}{2}\right)=-z(t), v\left(t+\frac{1}{6}\right)=-v(t)$. Column "\#” gives a series of reference numbers used for identification in the text.

\begin{tabular}{|c|c|c|c|c|}
\hline Type & $H$ generators & $K$ generators & Muscle oscillation & \# \\
\hline$(\mathbb{O}, \mathbb{T})$ & $V_{4}, F_{5}$ & $V_{4}, A_{3}$ & $(u(t),-u(t),-u(t), u(t))$ & 1 \\
\hline \multirow[t]{4}{*}{$\left(\mathbf{S}_{3}, \mathbf{Z}_{3}\right)$} & $V_{1}, E_{15}$ & $V_{1}$ & $(u(t), z(t), z(t),-z(t))$ & 2 \\
\hline & $V_{3}, E_{14}$ & $V_{3}$ & $(z(t), u(t),-z(t), z(t))$ & \multirow[t]{2}{*}{3} \\
\hline & $V_{5}, E_{16}$ & $V_{5}$ & $(z(t),-z(t), u(t), z(t))$ & \\
\hline & $V_{7}, E_{45}$ & $V_{7}$ & $(-z(t), z(t), z(t), u(t))$ & 4 \\
\hline \multirow[t]{6}{*}{$\left(\mathbf{D}_{2}^{E}, \mathbf{Z}_{2}^{E}\right)$} & $E_{16}, E_{15}$ & $E_{16}$ & $(u(t), u(t), 0,0)$ & \multirow[t]{2}{*}{$\overline{5}$} \\
\hline & $E_{14}, E_{13}$ & $E_{14}$ & $(u(t), 0, u(t), 0)$ & \\
\hline & $E_{45}, E_{35}$ & $E_{45}$ & $(0, u(t),-u(t), 0)$ & 6 \\
\hline & $E_{13}, E_{14}$ & $E_{13}$ & $(0, u(t), 0, u(t))$ & 7 \\
\hline & $E_{15}, E_{16}$ & $E_{15}$ & $(0,0, u(t), u(t))$ & \\
\hline & $E_{35}, E_{45}$ & $E_{35}$ & $(u(t), 0,0,-u(t))$ & 8 \\
\hline \multirow[t]{3}{*}{$\left(\mathbf{Z}_{4}, \mathbf{1}\right)$} & $F_{1}$ & $I$ & $\left(u(t), u\left(t+\frac{1}{4} \delta\right), u\left(t+\frac{3}{4} \delta\right), u\left(t+\frac{1}{2} \delta\right)\right)$ & 9 \\
\hline & $F_{3}$ & $I$ & $\left(u(t), u(t), u\left(t+\frac{1}{4} \delta\right), u\left(t+\frac{1}{4} \delta\right)\right)$ & \multirow[t]{2}{*}{10} \\
\hline & $F_{5}$ & $I$ & $\left(u(t), u\left(t+\frac{1}{4} \delta\right), u(t), u\left(t+\frac{1}{4} \delta\right)\right)$ & \\
\hline \multirow[t]{4}{*}{$\left(\mathbf{Z}_{3}, \mathbf{1}\right)$} & $V_{1}$ & $I$ & $\left(v(t), u(t), u\left(t+\frac{1}{3} \delta\right),-u\left(t+\frac{2}{3} \delta\right)\right)$ & 11 \\
\hline & $V_{3}$ & $I$ & $\left(u(t), v(t),-u\left(t+\frac{1}{3} \delta\right), u\left(t+\frac{2}{3} \delta\right)\right)$ & \multirow[t]{2}{*}{12} \\
\hline & $V_{5}$ & $I$ & $\left(u(t),-u\left(t+\frac{2}{3} \delta\right), v(t), u\left(t+\frac{1}{3} \delta\right)\right)$ & \\
\hline & $V_{7}$ & $I$ & $\left(u(t),-u\left(t+\frac{1}{3} \delta\right),-u\left(t+\frac{2}{3} \delta\right), v(t)\right)$ & 13 \\
\hline \multirow[t]{3}{*}{$\left(\mathbf{D}_{4}, \mathbf{Z}_{4}\right)$} & $F_{1}, A_{2}$ & $F_{1}$ & $(u(t), u(t), u(t), u(t))$ & $\overline{14}$ \\
\hline & $F_{3}, A_{3}$ & $F_{3}$ & $(u(t),-u(t), u(t),-u(t))$ & \multirow[t]{2}{*}{15} \\
\hline & $F_{5}, A_{1}$ & $F_{5}$ & $(u(t), u(t),-u(t),-u(t))$ & \\
\hline
\end{tabular}

while $y_{1}(t)=v(t)$ is independent of these. However, the same phase shifts apply to $y_{1}$, so we must have $v(t) \equiv v\left(t+\frac{1}{3} \delta\right)$. Moreover, like every periodic state arising by Hopf bifurcation, $v$ also satisfies $v\left(t+\frac{1}{2}\right)=-v(t)$. These observations lead to the condition $v\left(t+\frac{1}{6}\right)=-v(t)$ in the table.

Note that for phase shifts other than $0, \frac{1}{2}$ the states come in pairs, with plus or minus the stated phase shift. These pairs are identical except for time-reversal. For a given imaginary eigenspace, either one of these states occurs, or the other does, but not both. See [9, pp. 112-114]. (When $H / K \cong Z_{m}$ with $m=5$ or $m \geq 7$ the same pair $H / K$ can correspond to several distinct phase shifts, even taking the sign into account. For example, the $\mathbf{Z}_{5}$ case can have phase shift $\frac{2}{5}$ as well as $\frac{1}{5}$. However, these cases do not occur in the group $\mathbb{O}$.)

Table 4 lists (up to conjugacy) five small amplitude periodic state types that can occur by Hopf bifurcation supported by the standard 3-dimensional irreducible representation of $\mathbb{O}$, plus a sixth supported by the 1-dimensional representation. We interpret these motions in terms of our caricature of the muscle groups. We will see that all six cases lead to repetitive motions that seem quite reasonable.

Conjugate states are determined by $\mathbb{O} /(\boldsymbol{N}(\boldsymbol{H}) \cap \boldsymbol{N}(\boldsymbol{K}))$. We briefly discuss the technical issue: states whose associated subgroups are conjugate.

Suppose that $x(t)$ is a periodic state with spatiotemporal symmetry group pair $(H, K)$. Let $\gamma \in \Gamma$. Then $\gamma x(t)$ is a periodic state with spatiotemporal symmetry group pair $\left(H^{\prime}, K^{\prime}\right)$, where

$$
H^{\prime}=\gamma H \gamma^{-1} \quad \text { and } \quad K^{\prime}=\gamma K \gamma^{-1} .
$$


Thus the symmetry group pairs are identical if and only if $\gamma \in N(H) \cap N(K)$. The number of conjugate periodic states with different spatiotemporal symmetries is

$$
\frac{|\Gamma|}{|N(H) \cap N(K)|} .
$$

When we specialize to $\Gamma=\mathbb{O}$, the number of conjugates can be found by computing the normalizers of the appropriate subgroups. The normalizers are found in Table 2. In particular, $N(\mathbb{O}) \cap N(\mathbb{T})=\mathbb{O}, N\left(\mathbf{S}_{3}\right) \cap N\left(\mathbf{Z}_{3}\right)=\mathbf{S}_{3}, N\left(\mathbf{D}_{2}^{E}\right) \cap N\left(\mathbf{Z}_{2}^{E}\right)=\mathbf{D}_{2}^{E}$, $N\left(\mathbf{Z}_{4}\right)=\mathbf{D}_{4}, N\left(\mathbf{Z}_{3}\right)=\mathbf{S}_{3}$, and $N\left(\mathbf{D}_{4}\right) \cap N\left(\mathbf{Z}_{4}\right)=\mathbf{D}_{4}$. It follows that the number of conjugacies of the six solution types are $1,4,6,3,4,3$, respectively, yielding 21 possibilities.

6. Head motions. The standard equivariant theory classifies solution types up to conjugacy by a symmetry element. However, conjugate states are important here, because, with one exception, the action of $\mathbb{O}$ on the muscle space network does not relate directly to motions of the head in physical space $\mathbf{R}^{3}$, and that exception is the bilateral (left-right) symmetry of the body, which is realized in our network by $E_{45}$. So, in general, conjugate symmetry groups can correspond to head motions that are substantially different. Counting conjugates, as we have in Table 4, leads to 21 motions to describe -28 if we include time-reversals for \pm phase shifts. If we consider solution types up to time-reversibility and bilateral symmetry, then there are 15 types to consider. The final column (\#) in Table 4 is a reference number which we will use to identify the various patterns of oscillation.

Description of motions listed in Table 4. To explain the derivation of Table 4 , we take each conjugacy class in turn and visualize the corresponding periodic state in the following manner. We assume, for simplicity, a 2-dimensional description in which the head is modeled by a circle, as in Figure 6. The position of the neck is identified with the center of this circle. The orientation of the nose (under rotation about the neck axis) is specified by a vector based at the center of the circle with the appropriate orientation.

We decompose the head motion into two distinct components. The spatial motion of the head is obtained by summing the six vectors representing the muscle groups of the central dial. As time $t$ varies through a cycle, the resultant vector describes a closed curve in the horizontal plane, schematically representing the motion of the center of the circle that represents the head position.

Rotations of the neck (caused by muscle groups LA, RA) are represented as rotations of the circle about its instantaneous center. These rotations are assumed to act independently of the translations of the circle. This assumption is invalid in genuine 3-dimensional motion, but it provides an adequate visualization of small amplitude motions, bearing in mind that Figure 6 is itself a caricature.

Next, we choose specific periodic functions $u, z, v$ with the correct symmetry properties. For the figures drawn here we take

$$
\begin{aligned}
& u(t)=\sin (2 \pi t)+0.2 \sin (10 \pi t), \\
& z(t)=0.75 \sin (2 \pi t)+0.03 \sin (10 \pi t), \\
& v(t)=0.3 \sin (6 \pi t) .
\end{aligned}
$$

Then we use Table 4 to compute the six vectors of the central dial and the rotation angle of the nose. We denote the center of the circle (head) as a function of time $t$ by 


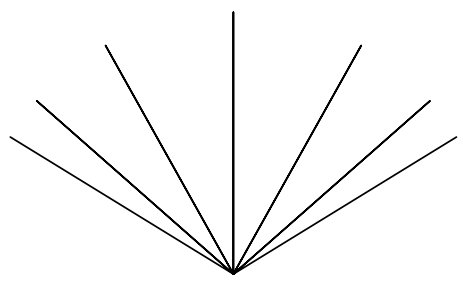

FIG. 8. Motion for pattern $1(\mathbb{O}, \mathbb{T})$.
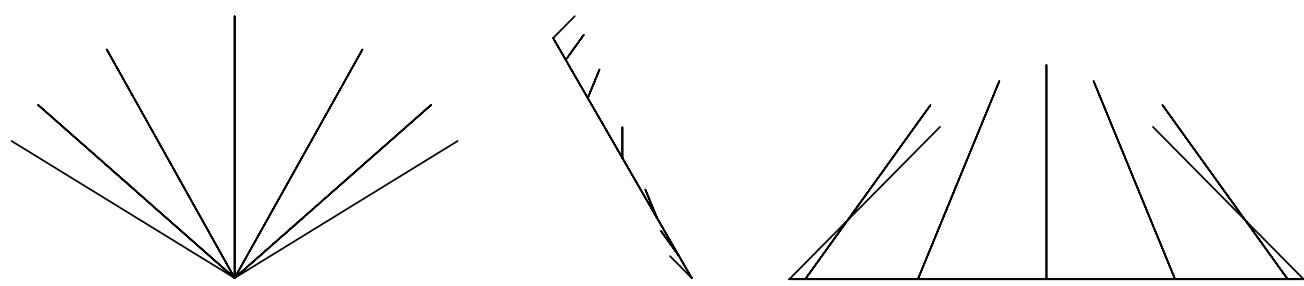

FIG. 9. Motion for patterns $2-4\left(\mathbf{S}_{3}, \mathbf{Z}_{3}\right)$.

the curve $\mathcal{C}(t)$. For a periodic state $y(t)=\left(y_{1}(t), y_{3}(t), y_{5}(t), y_{7}(t)\right)$ the closed curve $\mathcal{C}(t)$ has the form

$$
\begin{aligned}
& \mathcal{C}_{1}(t)=-2 y_{7}(t)-y_{3}(t)-y_{5}(t), \\
& \mathcal{C}_{2}(t)=\sqrt{3}\left(y_{3}(t)-y_{5}(t)\right) .
\end{aligned}
$$

The term $\sin (10 \pi t)$ is included to remove some artificial regularities from the pictures, such as motions of the head in a perfect circle. The vector representing the orientation of the nose is drawn at times $\frac{n}{12}$ for $0 \leq n \leq 11$, as a vector based on the appropriate point of the curve $\mathcal{C}$, of fixed length.

This representation involves some arbitrary choices, but is adequate for our present needs. When interpreting the figures, note that $\mathcal{C}$ may reduce to a line segment (described twice) or even a single point. Also, the segments representing the nose may overlap each other or overlap $\mathcal{C}$. These ambiguities can be resolved by creating a movie.

$(\mathbb{O}, \mathbb{T}):$ Here the muscles of the central dial follow the pattern $y_{3}(t)=y_{\mathrm{LF}}(t)=$ $-u(t), y_{5}(t)=y_{\mathrm{LE}}(t)=-u(t), y_{7}(t)=y_{\mathrm{LD}}(t)=u(t)$. From (6.1) we see that the curve $\mathcal{C}$ is a single point, and the center of the head does not move. The nontrivial head motion comes from $y_{\mathrm{LA}}$ and $y_{\mathrm{RA}}$, which swivel the head about its vertical axis. The overall invariance under $\left(-I, \frac{1}{2}\right)$ implies that this swivel motion is the same as its left-right reflection, up to a half-period phase shift. This description corresponds exactly, under the assumptions of the model, to the usual "shake the head" motion indicating the word "no." The schematic visualization of this motion is shown in Figure 8. Here the nose vector oscillates from left to right to form the fan shape illustrated.

$\left(\mathbf{S}_{3}, \mathbf{Z}_{3}\right) \therefore$ If we take $H=\left\langle V_{1}, E_{15}\right\rangle$ and $K=\left\langle V_{1}\right\rangle$, then this case turns out to be exactly like the previous one, except that the time series of the direct muscled motoneurons $u(t)$ is unequal to the time series of the central dial muscle motoneurons $z(t)$. (Here angle brackets indicate the subgroup generated by their contents.) Since the $z(t)$ motions cancel out, the motion again looks like "no" and is reproduced as the first image in Figure 9. 

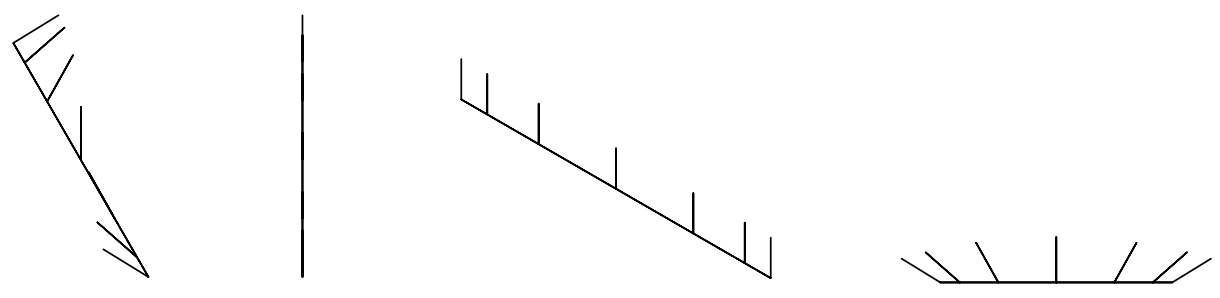

FIG. 10. Motion for oscillation patterns 5-8 ( $\left.\mathbf{D}_{2}^{E}, \mathbf{Z}_{2}^{E}\right)$.
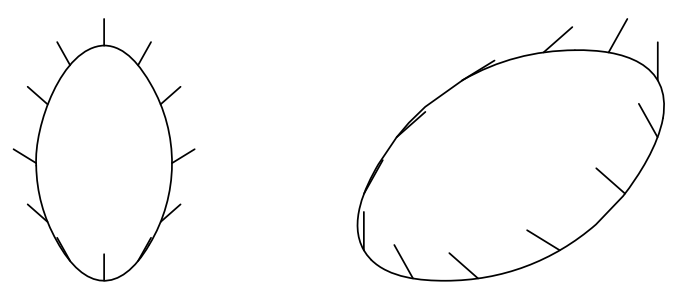

FIG. 11. Motion for patterns $9-10\left(\mathbf{Z}_{4}, \mathbf{1}\right)$.

The patterns for the other two conjugates of this motion can be deduced in a similar manner and are visualized in Figure 9. In pattern 3 the head is inclined alternately down to the left and up to the right, while the nose oscillates from side to side. In pattern 4 , the head tilts alternately to left and right while the nose oscillates from side to side.

$\left(\mathbf{D}_{2}^{E}, \mathbf{Z}_{2}^{E}\right)$ : First, we consider the conjugate state pattern 5 , for which $K=\left\langle E_{16}\right\rangle$, $H=\left\langle E_{16}, E_{15}\right\rangle$, and $y(t)=(u(t), u(t), 0,0)$. The phase shift action of $H / K$ implies that $u\left(t+\frac{1}{2}\right)=-u(t)$. Now the muscle groups LD, RD, LE, RF are inactive, LA and LF are in phase with each other, and RA and RE are half a period out of phase with LA and LF. The head "nods" down and to the left, then up and to the right, in roughly the direction of the muscle pair LF, RE, with a twist to the right as the head moves down, a twist to the left as it moves up. Another conjugate state has $K=\left\langle E_{14}\right\rangle$ and $H=\left\langle E_{14}, E_{13}\right\rangle$. This state is just the left/right image of the previous one.

Second, we consider pattern 6 , where $K=\left\langle E_{45}\right\rangle$ and $H=\left\langle E_{45}, E_{35}\right\rangle$. Such a state has $y_{\mathrm{LA}}=y_{\mathrm{RA}}=y_{\mathrm{LD}}=y_{\mathrm{RD}}=0$. The variables $y_{\mathrm{LF}}$ and $y_{\mathrm{LE}}$ are half a period out of phase, and the push-pull constraint implies that $y_{\mathrm{RF}}$ is in synchrony with $y_{\mathrm{LF}}$, and similarly $y_{\mathrm{RE}}$ is in synchrony with $y_{\mathrm{LE}}$. There is thus an overall leftright symmetry, and also a front-back symmetry when combined with a half period phase shift. This is precisely the pattern of movement observed when nodding the head (indicating "yes"). Motions associated with patterns 7 and 8 are found similarly. Note that pattern 8 also corresponds to a standard head motion: one where the head rotates left as it tilts left and then rotates right as it tilts right. See Figure 10 for diagrams of patterns $5-8$.

$\left(\mathbf{Z}_{4}, \mathbf{1}\right):$. We take $H=\left\langle F_{1}\right\rangle$. From Table 3 , and noting that $F_{1}$ induces a phase shift of $\pm \frac{1}{4}$, we obtain the pattern listed in Table 4 . (We also use the $\left(-I, \frac{1}{2}\right)$ symmetry of all periodic states.) The motions are visualized in Figure 11.

In pattern 9, the head moves in an ellipse with long axis pointing towards the front. The nose oscillates from side to side, moving outwards at the front and inwards at the back. There are two conjugates in pattern 10 that are mirror images of each 
other. The motion is much as above, but the ellipse is oriented along a different axis.

$\left(\mathbf{Z}_{3}, \mathbf{1}\right)$ : . Take $H=\left\langle V_{1}\right\rangle$. This leads to the pattern stated in Table 4. Bearing in mind the $\left(-I, \frac{1}{2}\right)$ symmetry, successive phases around the central dial differ by $\frac{1}{6}$. The head rotates in a "circle" (strictly, a closed loop with hexagonal symmetry), combined with a swivel. Choice of plus or minus phase shifts produce clockwise or counterclockwise rotations. Conjugates here replace $V_{1}$ by $V_{3}, V_{5}, V_{7}$, noting that $V_{3}$ and $V_{5}$ are mirror images. The motions are visualized in Figure 12. In pattern 11 the head rotates in a rounded hexagonal curve, while the nose oscillates slightly. The other two patterns are more complicated and best described using the figure.
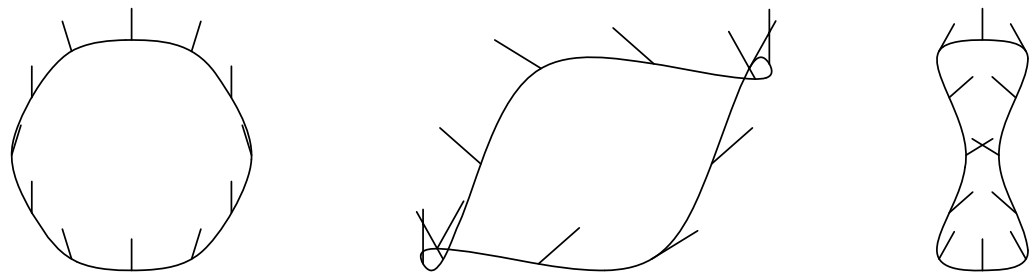

FIG. 12. Motion for patterns 11-13 $\left(\mathbf{Z}_{3}, \mathbf{1}\right)$.

$\left(\mathbf{D}_{4}, \mathbf{Z}_{4}\right) \therefore$. We choose $H=\left\langle F_{3}, A_{3}\right\rangle, K=\left\langle F_{3}\right\rangle$, and $y(t)=(u(t),-u(t), u(t),-u(t))$. The conjugates are as shown in Table 4 . The motions are visualized in Figure 13. In each case the head moves in one of three planes (so that $\mathcal{C}$ reduces to a line segment), while the nose oscillates from side to side. In pattern 14 the head moves left and right while the nose aims at a fixed central point.
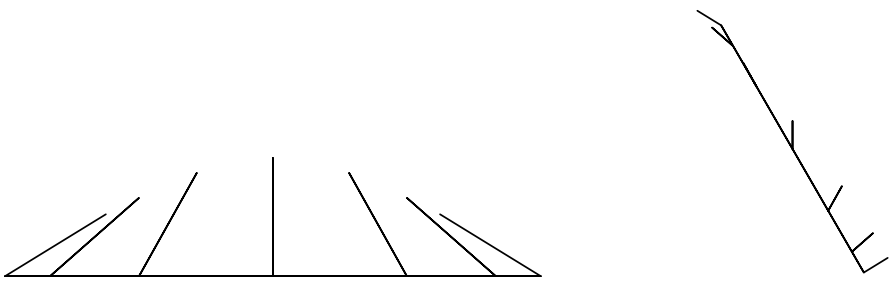

FIG. 13. Motion for patterns $14-15\left(\mathbf{D}_{4}, \mathbf{Z}_{4}\right)$.

7. Conclusions. In sections $2-4$ we derived the octahedral symmetry of the canal-neck projection first discovered by McCollum and Boyle [12]. After conjecturing that the symmetry of the network of neck muscle motoneurons also has octahedral symmetry, we classified the spatiotemporal symmetry types of small amplitude periodic solutions that can be obtained by Hopf bifurcation. Finally, in section 6 , we used the caricature of muscle group actions developed in section 2 to suggest the form that head motions might take.

On a cautionary note, the symmetries of neuronal networks need not reflect symmetries in the physical world. This mismatch in symmetry happens in the network associated with orientation-tuned neurons in the primary visual cortex [2] and is also the case in the vestibular system. Thus, periodic solutions that are symmetrically related in the network need not be (obviously) related in physical space (actual head movements). We believe that the issue of network structure not being directly related to physical world structure will be an important issue in many applications. 
The next steps in the program we have described are to include in the model the (symmetry) structure of other projections in the vestibular system, for example the uvula-nodulus [7], to include the semicircular canals, and to make more direct contact with the biology. Two questions arise in this last step: Do the head motions we describe play some special role in the context of general periodic head motions (that is, do these motions appear frequently in animals), and can the classification of spatiotemporal symmetries of small amplitude motions near an upright head give a method for classifying types of head tremor? Our classification, which is based on the symmetries of a network that has been abstracted from the neurobiology of the cat, provides a prediction for likely types of periodic head motions, much like the predictions that were implicit in our previous work on animal gaits [10,11].

There may exist periodic states that emanate from many types of bifurcation; however, in this study we classify only those types that emanate from a Hopf bifurcation.

Appendix: Characters of the octahedral group. The most efficient way to decompose the space $Y^{-}$into irreducible representations of $\mathbb{O}$ or $\mathbb{O} \oplus \mathbf{Z}_{2}^{c}$ is to use character theory; see, for example, Curtis and Reiner [6]. Recall that for a given representation of a group $\Gamma$, the corresponding character $\chi$ is the function $\chi: \Gamma \rightarrow \mathbf{C}$ for which $\chi(\gamma)$ is the trace of the matrix that represents the action of $\gamma \in \Gamma$. We assume familiarity with character theory.

First, observe that any representation (space) $U$ for $\mathbb{O}$ naturally determines two distinct representations $U^{+}, U^{-}$of $\mathbb{O} \oplus \mathbf{Z}_{2}^{c}$ with the same underlying vector space. In both, the elements of $\mathbb{O}$ have the same action as they do on $U$. The action of $-I$ on $U^{+}$is by the identity, whereas that on $U^{-}$is by minus the identity. If $U$ is irreducible for the action of $\mathbb{O}$, then the $U^{ \pm}$are irreducible for the action of $\mathbb{O} \oplus \mathbf{Z}_{2}^{c}$.

It is easy to prove that every irreducible for $\mathbb{O} \oplus \mathbf{Z}_{2}^{c}$ arises in this manner, as follows. Every irreducible representation of $\mathbb{O}$ is absolutely irreducible, so by Schur's lemma the only commuting linear maps are scalar multiples of the identity. Since $-I$ commutes with $\mathbb{O}$ and $(-I)^{2}=I$, it follows that $-I$ must act as plus or minus the identity. The rest is straightforward.

Therefore we can read off the irreducible representations of $\mathbb{O} \oplus \mathbf{Z}_{2}^{c}$ from those of (O). It is well known (see, for example, Curtis and Reiner [6, pp. 331-333]) that $\mathbb{O}$ has five distinct irreducible representations: two of dimension 1 , one of dimension 2 , and two of dimension 3 .

We can describe the irreducible representations of $\mathbb{O}$ as follows:

- $\rho_{0}$ : dimension 1 ; trivial action.

- $\rho_{1}$ : dimension $1 ; \mathbb{T}$ acts trivially, $\mathbb{O} \backslash \mathbb{T}$ acts by -1 .

- $\rho_{2}$ : dimension 2 ; kernel is the Klein four-group $\mathbf{D}_{2}^{A}$, modulo which $\mathbb{O}$ acts in the standard representation of $\mathbf{D}_{3}$ on $\mathbf{R}^{2}$.

- $\rho_{3}$ : dimension 3; standard action of $\mathbb{O}$ as isometries of $\mathbf{R}^{3}$ preserving the cube.

- $\rho_{4}$ : dimension 3 ; nonstandard action on $\mathbf{R}^{3}$ in which $\mathbb{T}$ acts as rotations but $\mathbb{O} \backslash \mathbb{T}$ acts as rotations composed with minus the identity. In fact, $\rho_{4}=\rho_{1} \otimes \rho_{3}$. This representation is also isomorphic to the standard action of $\mathbf{S}_{4}$ on the subspace of $\mathbf{R}^{4}$ consisting of points whose coordinates sum to 0 .

The conjugacy classes of $\mathbb{O}$ are also five in number. In the notation of Table 3 they are

$$
\{I\}, \quad\left\{A_{j}\right\}, \quad\left\{V_{j}\right\}, \quad\left\{F_{j}\right\}, \quad\left\{E_{j}\right\} .
$$


The character table for $\mathbb{O}$ is shown in Table 5 , and is derived in Curtis and Reiner [6, pp. 332-333]. It is easy to compute the character $\chi$ of the $\mathbb{O}$-action described in Table 3, which is shown in Table 5 in the same format. In particular, we see that $\chi=\rho_{1}+\rho_{3}$. Since characters determine representations uniquely, and direct sums of representations correspond to sums of characters, we see that $Y^{-}$decomposes into two irreducible components, the nontrivial 1-dimensional representation and the standard 3 -dimensional representation. This is what we claimed in section 4.

TABLE 5

Character table for representations of $\mathbb{O}$.

\begin{tabular}{|r||r|r|r|r|r|}
\hline & $\{I\}$ & $\left\{A_{j}\right\}$ & $\left\{V_{j}\right\}$ & $\left\{F_{j}\right\}$ & $\left\{E_{j}\right\}$ \\
\hline \hline$\rho_{0}$ & 1 & 1 & 1 & 1 & 1 \\
\hline$\rho_{1}$ & 1 & 1 & 1 & -1 & -1 \\
\hline$\rho_{2}$ & 2 & 2 & -1 & 0 & 0 \\
\hline$\rho_{3}$ & 3 & -1 & 0 & 1 & -1 \\
\hline$\rho_{4}$ & 3 & -1 & 0 & -1 & 1 \\
\hline \hline$\chi$ & 4 & 0 & 1 & 0 & -2 \\
\hline
\end{tabular}

Acknowledgments. We thank Gin McCollum, Patrick Roberts, Douglas Hanes, David Romano, and Paul Matthews for helpful discussions. We also thank the Newton Institute, University of Cambridge, and the Department of Mathematics, University of Toronto, for their hospitality.

\section{REFERENCES}

[1] P. Ashwin and O. Podvigina, Hopf bifurcation with cubic symmetry and instability of ABC flow, Proc. R. Soc. Lond. A Math. Phys. Eng. Sci., 459 (2003), pp. 1801-1827.

[2] P. C. Bressloff, J. D. Cowan, M. Golubitsky, P. J. Thomas, and M. C. Wiener, Geometric visual hallucinations, Euclidean symmetry, and the functional architecture of striate cortex, Phil. Trans. Royal Soc. London B, 356 (2001), pp. 299-330.

[3] P. L. Buono And M. Golubitsky, Models of central pattern generators for quadruped locomotion: I. Primary gaits, J. Math. Biol., 42 (2001), pp. 291-326.

[4] J. J. Collins and I. Stewart, Hexapodal gaits and coupled nonlinear oscillator models, Biol. Cybern., 68 (1993), pp. 287-298.

[5] J. J. Collins AND I. STEWART, Coupled nonlinear oscillators and the symmetries of animal gaits, J. Nonlinear Sci., 3 (1993), pp. 349-392.

[6] C. W. Curtis and I. Reiner, Representation Theory of Finite Groups and Associative Algebras, Wiley-Interscience, New York, 1962.

[7] I. Z. Foster, D. A. Hanes, N. H. Barmack, and G. McCollum, Spatial symmetries in vestibular projections to the uvula-nodulus, Biol. Cybernet., 96 (2007), pp. 439-453.

[8] M. Golubitsky and I. N. Stewart, Hopf bifurcation in the presence of symmetry, Arch. Ration. Mech. Anal., 87 (1985), pp. 107-165.

[9] M. Golubitsky And I. Stewart, The Symmetry Perspective, Progr. Math. 200, BirkhäuserVerlag, Basel, 2002.

[10] M. Golubitsky, I. Stewart, P.-L. Buono, and J. J. Collins, A modular network for legged locomotion, Phys. D, 115 (1998), pp. 56-72.

[11] M. Golubitsky, I. Stewart, P.-L. Buono, and J. J. Collins, Symmetry in locomotor central pattern generators and animal gaits, Nature, 401 (1999), pp. 693-695.

[12] G. MCCOllum AND R. Boyle, Rotations in a vertebrate setting: Evaluation of the symmetry group of the disynaptic canal-neck projection, Biol. Cybern., 90 (2004), pp. 203-217.

[13] Y. Shinoda, Y. Sugiuchi, T. Futami, N. Ando, And T. KaWASAKi, Input patterns and pathways from six semicircular canals to motoneurons of neck muscles I: The multifidus muscle group, J. Neurophysiol., 72 (1994), pp. 2691-2702.

[14] Y. Shinoda, Y. Sugiuchi, T. Futami, N. Ando, And J. Yagi, Input patterns and pathways from six semicircular canals to motoneurons of neck muscles II: The longissimus and semispinalis muscle groups, J. Neurophysiol. 72 (1997), pp. 2691-2702. 
[15] Y. Shinoda, Y. Sugiuchi, T. Futami, S. Kakei, Y. Izawa, and J. Na, Four convergent patterns of input from the six semicircular canals to motoneurons of different neck muscles in the upper cervical cord, Ann. New York Acad. Sci., 781 (1996), pp. 264-275.

[16] V. J. Wilson AND M. Maeda, Connections between semicircular canals and neck motoneurons in the cat, J. Neurophysiol., 37 (1974), pp. 346-357. 